\title{
Magnetohydrodynamic Turbulence at High-Latitude Regions of the Milky Way
}

\author{
Nickolas Pingel, Snežana Stanimirović, Min-Young Lee \\ University of Wisconsin-Madison ${ }^{1}$
}

\begin{abstract}
We performed statistical analysis on the high-latitude molecular cloud MBM16 with data collected from the Galactic Arecibo L-Band Feed Array HI (GALFA-HI) Survey. We developed a code to convolve a circular kernel across a spatial column density image to create maps of the $3^{\text {rd }}$ and $4^{\text {th }}$ statistical moments. With these higher-order moment maps, we utilized a relationship derived purely from the theoretical MHD simulations of Burkhart et al. (2010) to directly estimate the sonic Mach number $\left(\mathrm{M}_{\mathrm{S}}\right)$. We then looked at an estimate for the slope of the spatial power of our region to check our range of calculated Mach numbers and verify the reliability of our results. We found that the translucent, non-star forming molecular cloud, MBM16, can be considered largely subsonic to transonic, which is consistent with the conception of stars being dominant drivers of interstellar turbulence.
\end{abstract}

\section{Introduction}

Throughout the last decade, there has been much progress in both observations and theoretical models characterizing the evolution and overall workings of the interstellar medium (ISM). It is evident that interstellar turbulence plays an important role in ISM structure formation and evolution. While there have been many advances in understanding the functionality of turbulence within the ISM, many questions remain open about exactly how turbulence influences processes within the ISM. For instance, what are the prominent energy sources and physical processes that induce turbulence? At what scales and through which modes does turbulence energy dissipate in the ISM? How do different characteristics about the interstellar gas (e.g., presence/absence of star formation, strength of magnetic field, presence/absence of tidal effects) affect the amount and type of turbulence exhibited? These are difficult questions to answer in the absence of a complete astrophysical theory describing turbulence, which is why an approach combining numerical techniques and observations is required.

To address some of these questions, specifically about the presence or absence of star formation drives turbulence, we first looked at the numerical isothermal simulations of HI gas developed by Burkhart et al. 2010 (hereby referred to as BHK) to aid in a numerical approach. Our study is the first attempt for the Milky Way to study the spatial distribution of turbulence and will be extended to other clouds. We used a simple relationship between higher-order moments of column density distributions and the sonic Mach number to

\footnotetext{
${ }^{1}$ We acknowledge the Wisconsin Space Grant Consortium for providing funding for this research.
} 
determine how turbulence is disseminated across the cloud. We compared our results of our non-star forming cloud to BHK's estimate for turbulence for the Small Magellanic Cloud (SMC). This is important because star formation is present in the SMC, which enabled us to test the idea of stars being dominant drivers of turbulence. Finally, we tested the reliability of results by comparing our estimates of turbulence with an independent measure of turbulence for our region by Chepurnov et al. 2010.

In particular, this paper is organized as follows. We begin section 2 by giving a brief background on the data used to create the column density distribution maps used by our code to ultimately estimate the spatial distribution of turbulence in MBM16, and also highlight the important properties of this cloud. In section 3 we introduce the higher-order statistical moments used in this study, and explain their relationship to the sonic Mach number. We then explain how our code uses this relationship to create a sonic Mach number map, which describes the spatial distribution of turbulence in a region or cloud. In section 4 we present our results and compare them to BHK's work on the SMC, and test the reliability of our results by examining the slope of the power law of our region's spatial power spectrum. Lastly, in section 5, we discuss our results and discuss future works.

\section{The GALFA-HI Survey and MBM16}

Background on GALFA-HI. The GALFA-HI survey is a high-resolution, (4') largearea $\left(13,000 \mathrm{deg}^{2}\right)$, survey conducted at the Arecibo radio telescope in Puerto Rico using Arecibo's ALFA receiver and the GALSPECT spectrometer. ALFA is a seven-element array primarily designed for $21 \mathrm{~cm}$ observations. GALSPECT is a special-purpose spectrometer with a spectral resolution of $0.18 \mathrm{~km} \mathrm{~s}^{-1}$ used for Galactic science in conjunction with ALFA. Data were reduced with the standard GALFA-HI reduction strategy detailed in Peek et al. (2007) and observed in a "basket-weave" mode, interlacing scans from day to day. All of the data cubes used in this study are part of a general data release available for public use at http://purcell.ssl.berkeley.edu/ with specific details outlined in Peek et al. (2011).

Approximately $82 \%$ of the survey data collected has been obtained while operating communally with other large survey projects such as ALFALFA. This is a unique strength, with which multiple backends can be used in parallel, enabling different science projects to use the same telescope pointing simultaneously. Communal observing is especially powerful for a sky survey, and will be an imperative observation strategy with future generations of radio telescopes, most notably the SKA (Square Kilometer Array).

Properties of MBM16. The translucent, non-star forming molecular cloud, MBM16 (Figure 1), was first cataloged by Magnani, Blitz \& Mundy (1985) and has an estimated distance of about $80 \mathrm{pc}$ (Hobbs et al 1988). We chose to look specifically in high-latitude regions because the HI gas is considered less "contaminated" than $\mathrm{HI}$ in the plane-meaning there is a more pristine turbulent environment due to the lack of star formation. The relatively short distance to this cloud gives us a good spatial resolution of about $0.1 \mathrm{pc}$. The absence of stellar activity in MBM16 presented us with the opportunity to directly compare our results with BHK's work on the SMC, a star forming dwarf galaxy. 


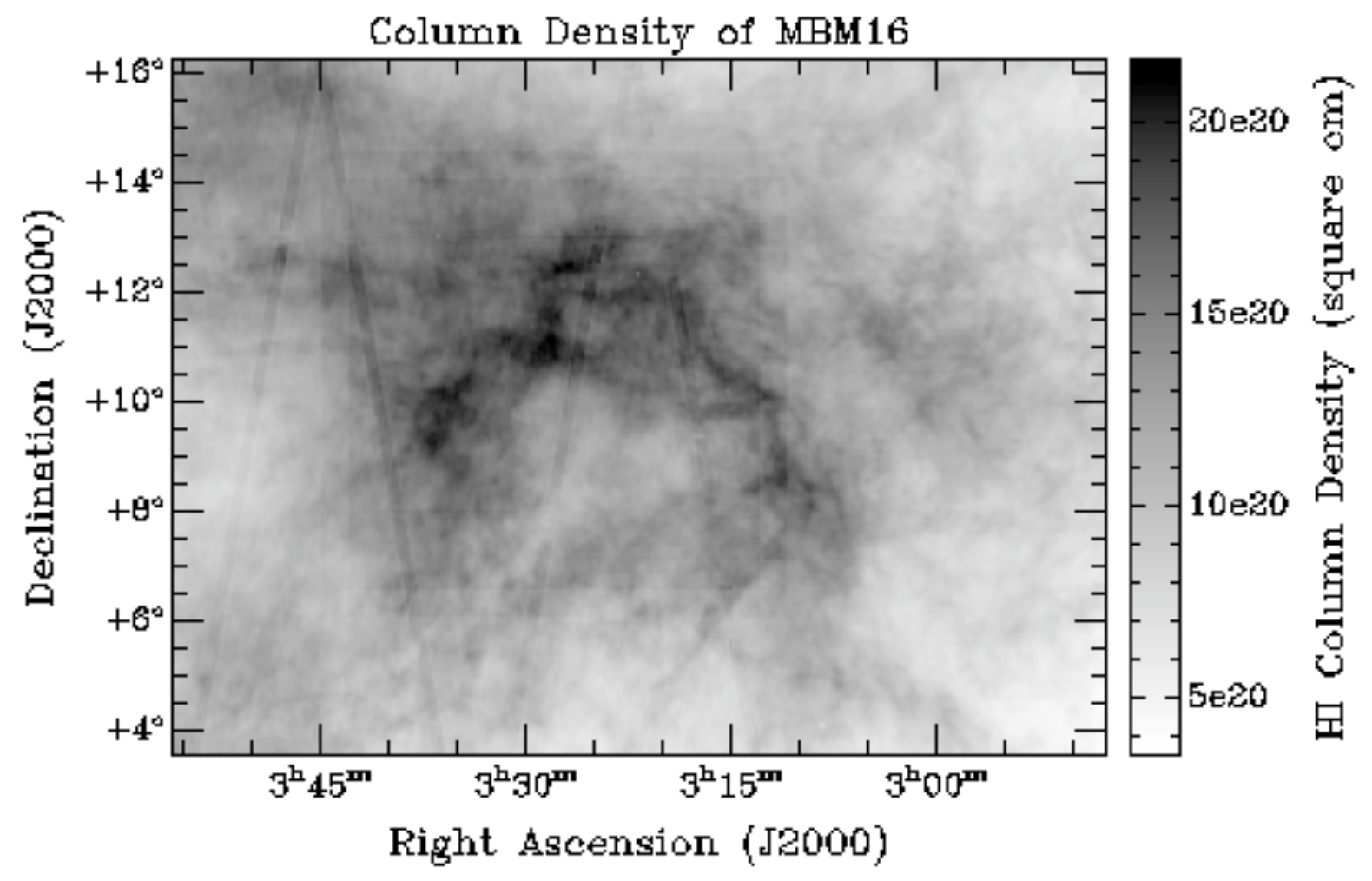

Figure 1-A column density map of MBM16. The lines through the image are remnants of the telescope observing pattern; data reduction techniques are still being modified.

\section{The Statistical Moments Used in this Study, Their Relationship with Turbulence and the Sonic Mach Number Map.}

Skewness and kurtosis. The two simplest properties of a distribution are the mean value and variance. While these are not directly used in this study, they are still required to calculate skewness and kurtosis - the more applicable statistical properties of our study. Skewness is defined as:

$$
\gamma_{\xi}=\frac{1}{N} \sum_{i=1}^{N}\left(\frac{\xi_{i}-\bar{\xi}}{\sigma_{\xi}}\right)^{3}
$$

Where $\sigma$ is the variance of the distribution $\xi$ of column density values and $\mathrm{N}$ is the number of samples. Skewness measures how skewed towards high or low data values a distribution is. Kurtosis is defined as:

$$
\beta_{\xi}=\frac{1}{N} \sum_{i=1}^{N}\left(\frac{\xi_{i}-\bar{\xi}^{4}}{\sigma_{\xi}}\right)-3
$$

Kurtosis measures how "peaked", or bunched a data set is. If a distribution has positive kurtosis, the probability density function (PDF) will have a distinct peak near the mean and elongated tails. Conversely, a negative kurtosis value will be flat at the mean. 
Relationship to $\mathbf{M}_{\mathbf{s}}$. BHK found that variance, skewness and kurtosis of their simulated column density distribution depended on turbulent properties, specifically the sonic Mach number. Figure 2 describes the correlation revealed by their simulations.
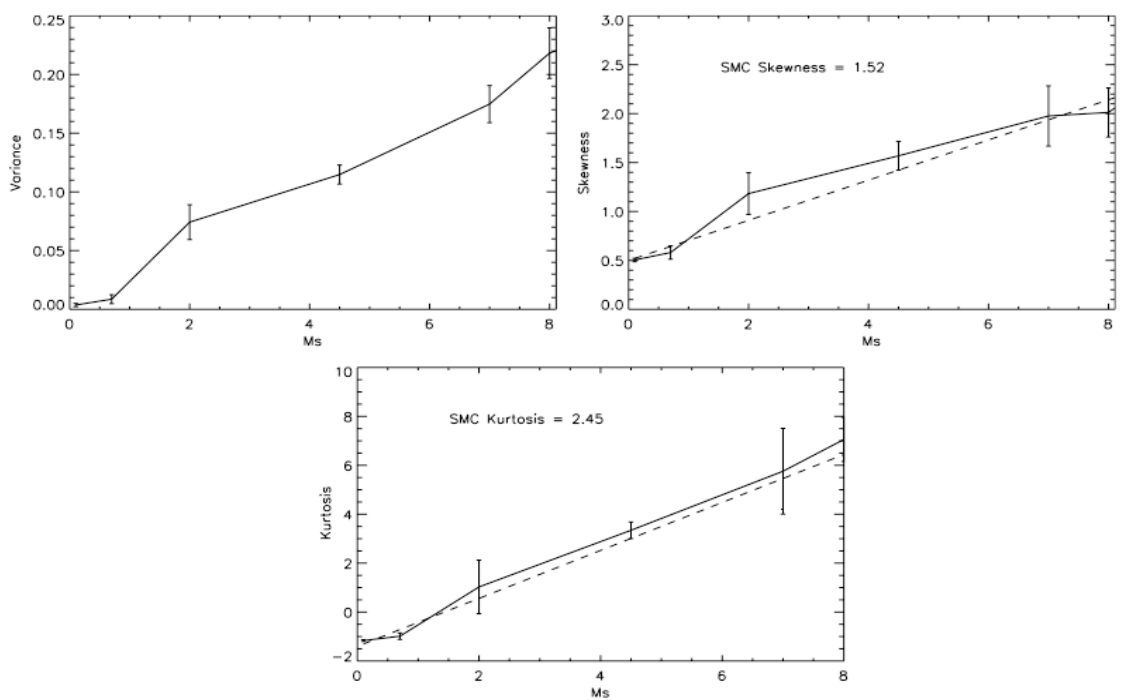

Figure 2-Taken from BHK. Skewness (top right) and kurtosis(bottom) show the best correlations between the higher-ordered moments, though kurtosis exhibits the simplest linear fit.

In order to develop these relationships, BHK essentially fixed the sonic Mach number for each numerical isothermal simulation and looked for a relationship between the higher-order moments and $\mathrm{M}_{\mathrm{s}}$. They were able to determine that the simple equation: $\mathrm{M}_{\mathrm{s}}=($ Kurtosis+1.44)/1.05 effectively estimates the Mach number of a distribution. In essence, the increase of higher order moments with $\mathrm{M}_{\mathrm{s}}$ can be explained in that as Mach number increases, the amount of small-scale structure increases, resulting in a broader PDF.

Determining the spatial distribution of skewness and kurtosis. In order to characterize small-scale departures from Gaussianity of the column density distributions across MBM16, we first had to spatially calculate skewness and kurtosis. To accomplish this, we developed a code that convolves the column density image with a circular kernel with a given radius $r$ that calculates skewness and kurtosis at all points. BHK decided on a kernel with a radius of $r=35$ pixels to maximize signal-tonoise. We should note that the column density image of MBM16 was scaled using the standard score method to be consistent with the procedure from BHK.

Our code begins this process by creating a box kernel in the lower left corner of the column density image in Figure 1. It then focuses on the central pixel of the box, and traces a circle with a radius of 35 pixels. Any pixel of the column density image within that circle is considered part of the distribution, which is multiplied by a Gaussian function to give more weight to the central pixels to minimize edge effects. Statistics are then run on the distribution within the circular kernel, calculating skewness and kurtosis at all points. The single value calculated for skewness and kurtosis then replaces all column density values within the circular kernel to start building the respective moment maps. The box kernel then moves to the right one pixel, and this 
process is repeated until it reaches the right edge; it will then elevate itself one pixel and begin again on the left edge. It should be noted that this is similar to convolving the image with a Gaussian function with a FWHM of 30' giving the resultant maps a lower spatial resolution of $\sim 0.7 \mathrm{pc}$. We find that MBM16 and surrounding mapped region has a kurtosis value of 2.45 and a skewness value of 1.57. The resulting moment map for kurtosis is displayed in Figure 3.

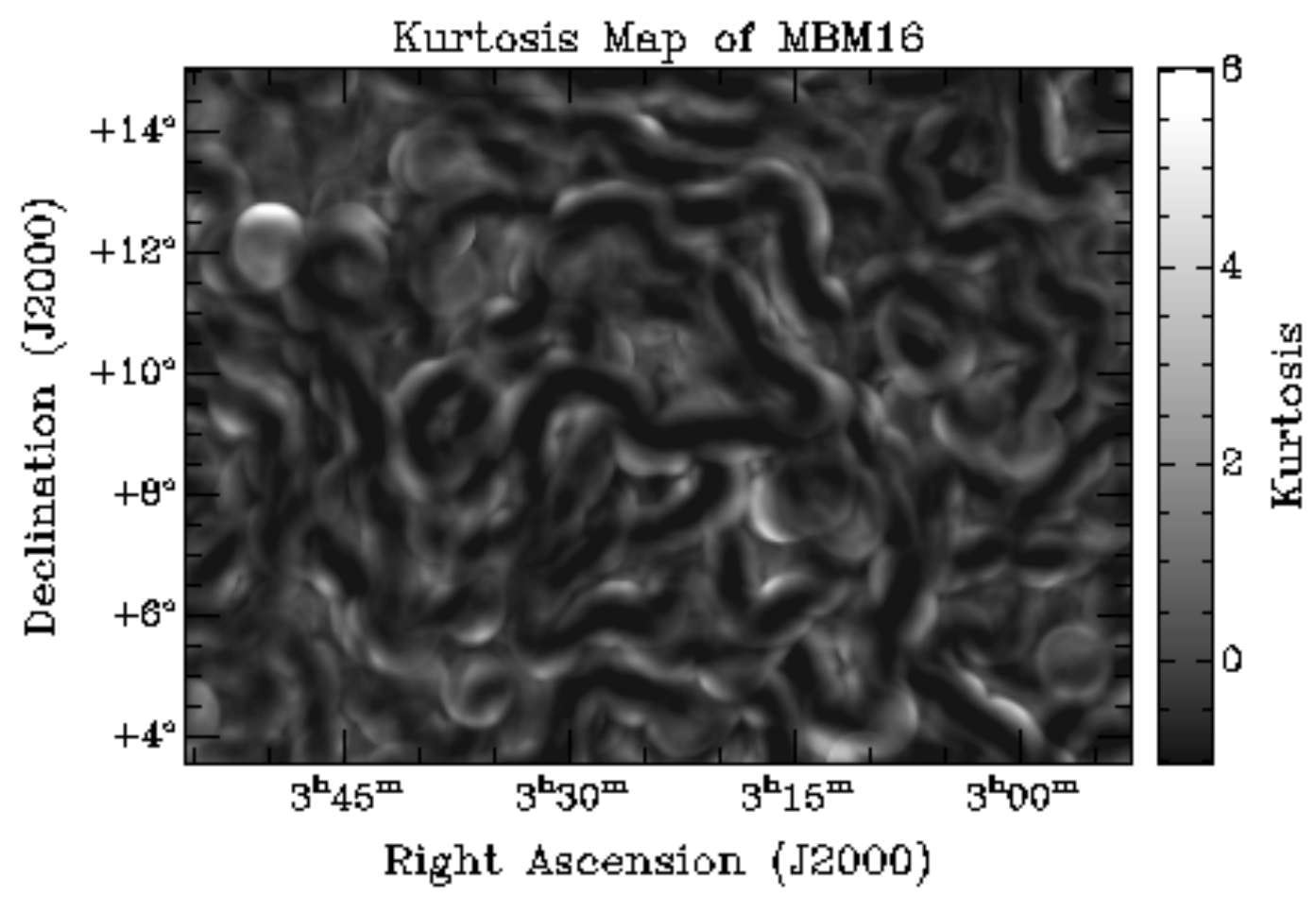

Figure 3-Kurtosis map. The moment maps are only intermediate steps to the final goal of a Sonic Mach number map. It is difficult to discern any spatial information from these maps, but we show them to highlight the raw output of our code.

Kurtosis has the best correlation to $\mathrm{M}_{\mathrm{s}}$ so we focused exclusively on the kurtosis map to create the end product of a Mach number map. To do this we took each pixel's value on the kurtosis map and put it into the simple equation: $\mathrm{M}_{\mathrm{s}}=($ kurtosis +1.44$) / 1.05$. The end product of a sonic Mach number map is presented in Figure 4. 


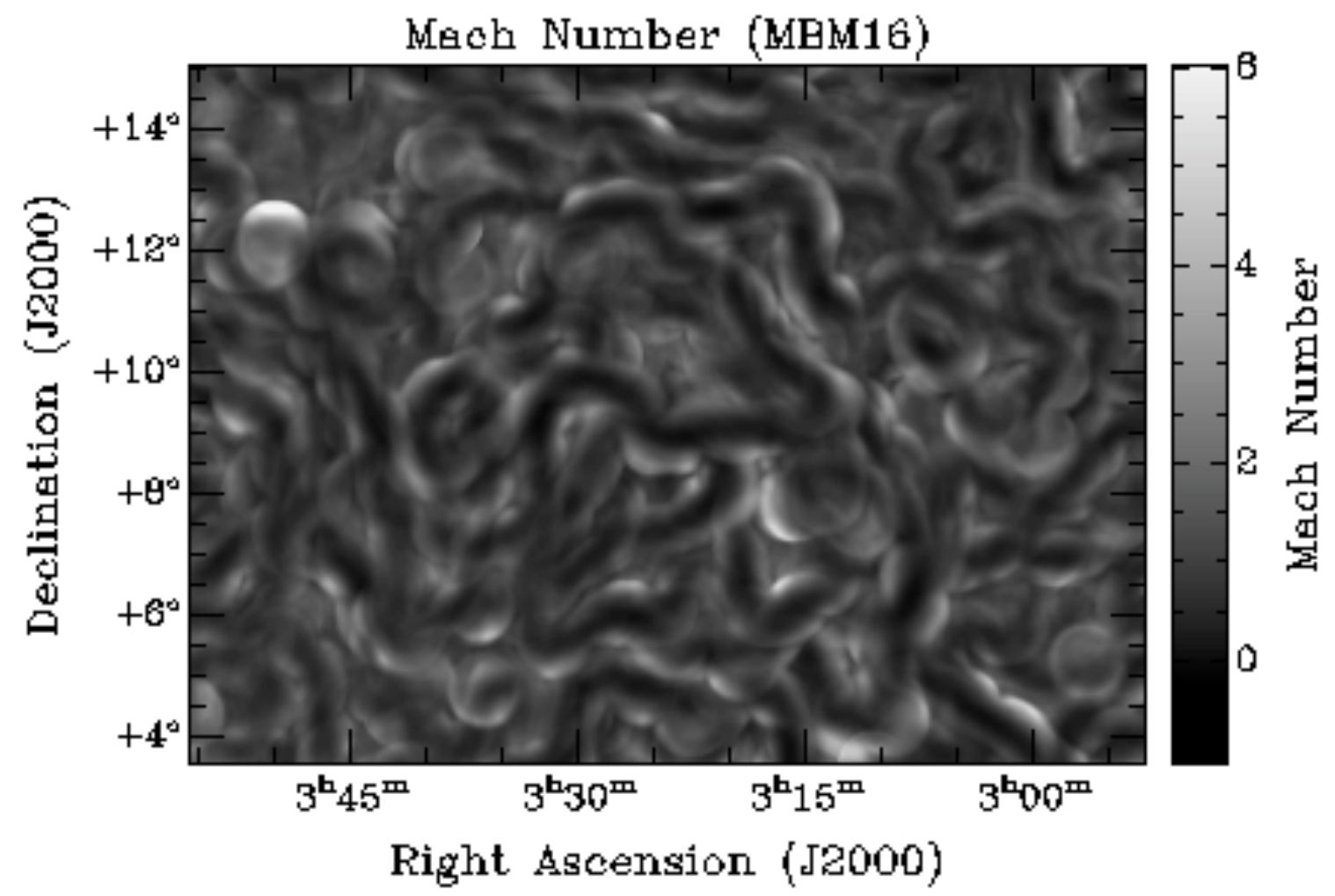

Figure 4-The Sonic Mach Number map of MBM16. This is the final product of our statistics code and highlights the spatial distribution of turbulence in MBM16.

\section{Results and Interpretation of Statistics}

Spatial distribution of turbulence. Though the sonic Mach number map is the targeted end product, it is not entirely effectual in highlighting the spatial relationship between column density distributions and sonic Mach numbers across the cloud. To accentuate any associations, we overlaid higher levels of mach numbers in Figure 5, along with smoothed HI contours. 


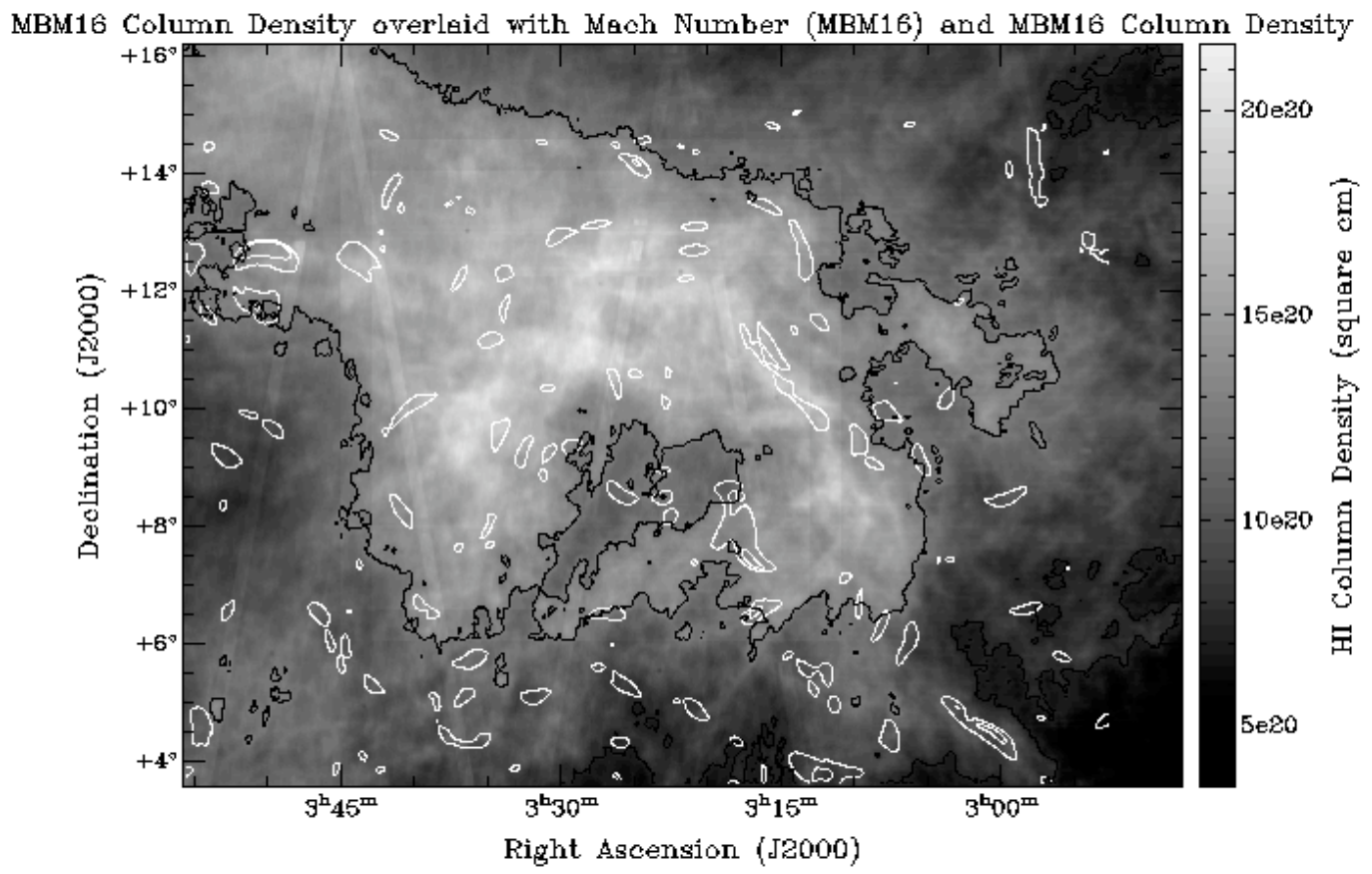

Figure 5-The original column density map of MBM16 with higher-level mach numbers overlaid with white contours, and smoothed HI contours in black. We see the most turbulent regions are small and localized compared to the entire cloud.

These contours show a few localized regions of high turbulence, and most of these regions are associated with an HI contour line. An interesting feature is a semi-circular shaped turbulent region located in the top left corner of the image at coordinates of $\mathrm{RA}=3 \mathrm{~h} 50 \mathrm{~m}$, $\mathrm{DEC}=12.30^{\circ}$. It is easily the most conspicuous turbulent region across the cloud, which enticed us for further examination. We examined the velocity space of the original data cube used to generate the column density map, but could not identify a physical process that we could associate with this feature. Nonetheless, it is very intriguing. We concluded that, as a whole, MBM16 is not turbulent expect for a few small localized regions.

Star forming vs. non-star forming environments. BHK developed the method used in this study, and first tested it on observations of the SMC, a dwarf satellite galaxy of the Milky Way. To test the notion of stars being major drivers of turbulence, we compared our results of our non-star forming cloud to BHK's results for the star forming SMC in Table 1, and in Figure 6 with histograms and plots of skewness vs. kurtosis. 


\begin{tabular}{lll}
$\mathbf{M}_{\mathbf{s}}$ & SMC & MBM16 \\
$0-1$ & $45 \%$ & $55 \%$ \\
$1-2$ & $45 \%$ & $39 \%$ \\
$>2$ & $10 \%$ & $6 \%$ \\
\hline
\end{tabular}

Table 1-Comparision of the turbulence across the mapped areas of MBM16 and the SMC.
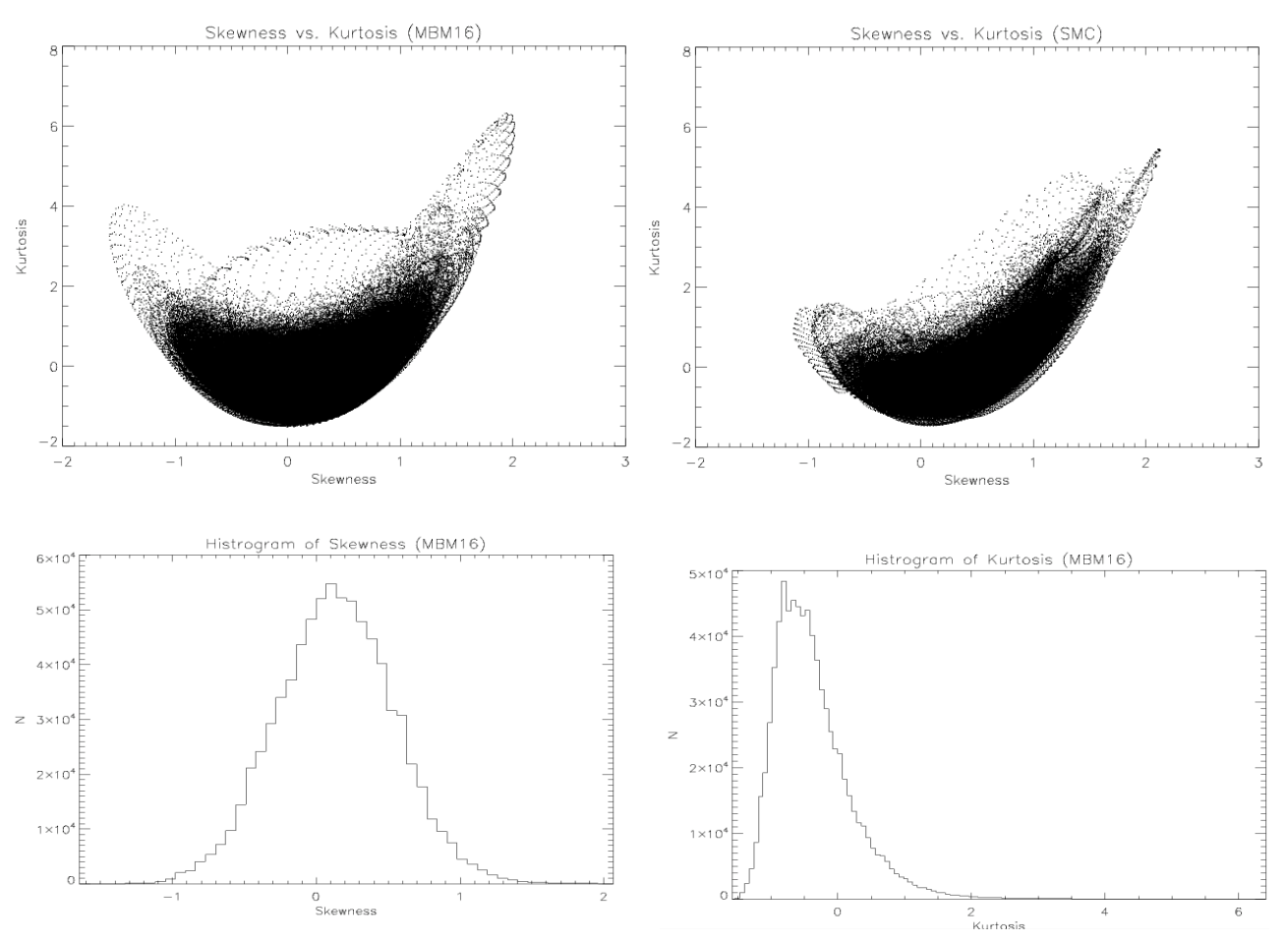

Figure 6- Skewness vs. Kurtosis plots of MBM16(left) and of the SMC(right). Below are histograms of skewness(left) and kurtosis(right) of MBM16.

Table 1 shows how supersonic $\left(M_{s} \geq 2\right)$ the SMC and MBM16 are by the percentage of their mapped area. MBM16 is less turbulent in every $\mathrm{M}_{\mathrm{s}}$ bin with just over half of the mapped area being subsonic $\left(M_{s} \leq 1\right)$ and only $39 \%$ being transonic $\left(1 \leq M_{s} \leq 2\right)$, while $10 \%$ of the SMC is supersonic to just $6 \%$ of MBM16. BHK found if there is a good correlation (i.e. linear relationship) between high levels of skewness and kurtosis, this region has non-gaussianities in the column density distributions, which means the region is more turbulent. The skewness vs. kurtosis plots at the top of Figure 6 shows this linear relationship between the higher values of the two moments for the SMC, but is not as pronounced in MBM16. We see why there should not be a good correlation by examining MBM16's histograms of skewness and kurtosis at the bottom of Figure 6. Skewness has values falling between -1 and 1, while most of the kurtosis values fall between 0 and -2 . With no obvious linear relationship, we 
concluded that the MBM16 is less turbulent than the SMC, which we expected due to the vast differences in stellar environments.

Testing the reliability of our results. BHK tested the dependability of their results by examining the slope of the spatial power spectrum for the SMC. We used the same strategy by examining Figure 7.

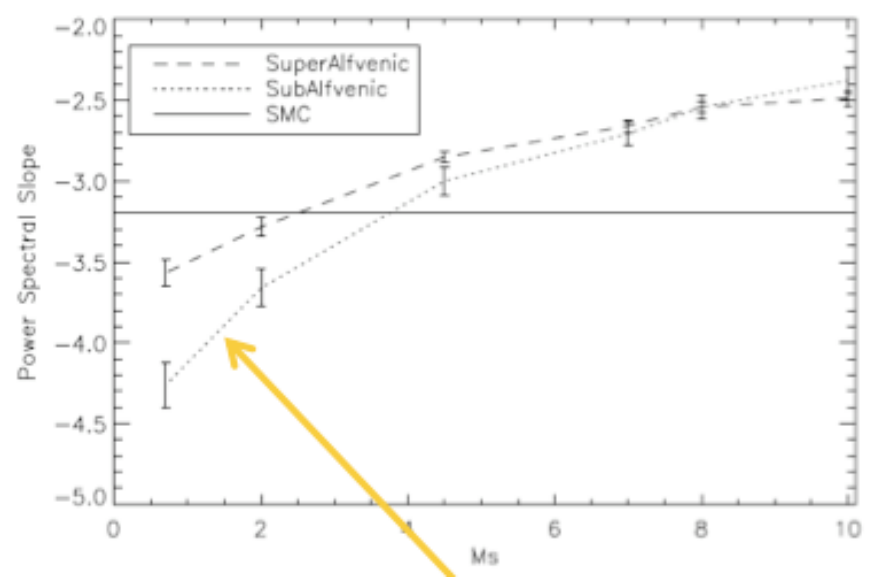

Figure 7- Taken from BHK. This plot is the power spectral slope as a function of Mach number for BHK's simulations. The solid horizontal line shows the SMC, while the arrow points to the measure slope for our region of -3.9 .

Chepurnov et al. (2010) measured the power spectral slope of our region to be -3.9. The basic concept of a spatial power spectrum is to quantify how much small scale structure exists; the steeper the slope, the less turbulent energy has cascaded down from large scales and mixed up the gas on small scales. A slope as steep as 3.9 indicates Mach numbers of around 2 and lower, meaning we should not expect to see much small scale structure across MBM16. This is because such low Mach numbers will not allocate enough turbulent energy to cascade down to the smaller scales. Our estimated range of Mach numbers is consistent with the power spectral slope measured by Chepurnov et al. (2010), and enforces our results.

\section{Discussion and Future Works.}

In this study we applied a statistical method on Galactic observations for the first time developed by BHK to estimate turbulence. We found that MBM16 can be characterized as a mostly subsonic to transonic with a few localized regions of high turbulence. We then compared our results with BHK's SMC work, and found MBM16 to be less turbulent than the SMC. Considering the absence of star formation in MBM16, we concluded that MBM16 is less turbulent than many Galactic regions, which is consistent with the notion of stars being dominant drivers of interstellar turbulence. We also found that our estimate for the range of mach numbers for MBM16 agree with the range indicated by the independently measured power spectrum slope by Chepurnov et al. 2010.

In a paper that is in preparation we will obtain our own estimate of the spatial power spectrum slope of MBM16 and compare it with the estimate of Chepurnov et al. 2010. We will also examine the spectral information of MBM16 in an attempt to better understand its 
velocity components. We plan to obtain an observational estimate of the sonic Mach number by examining background sources to see if any comparisons can be made to the results of the method used in this study. We can apply this method to the many cataloged MBM clouds within the GALFA-HI data, which will allow us to investigate how turbulence affects different environments and properties of interstellar gas. There is a plethora of CO data for MBM16, enabling us to use this statistical method on $\mathrm{CO}$ column density maps. It is intriguing to see what kinds of correlations and relationships may arise between the HI and $\mathrm{CO}$ data. These are preliminary results, which will quickly lead to a comprehensive paper.

We are very thankful for the NASA Wisconsin Space Grant Consortium for the generous funding of this research.

\section{References.}

Burkhart, B., Stanimirovic, S., Lazarian, A., \& Kowal, G. 2010, ApJ, 708, 1204

Chepurnov, A., Lazarian, A., Stanimirovic, S. , Heiles, C., \& Peek, J.E.G., 2010. ApJ, 714, 1398

Hobbs, L.M., Blitz, L., Penprase, B.E., Magnani, L., Welty, D.E., 1988, ApJ, 327, 356

Magnani, L.,Blitz, L, \& Mundy, L. 1985, ApJ, 402,295(MBM)

Peek J.E.G., Heiles, C.,Douglas, K.A., Lee, M.Y., Grecevich,

J., Stanimirovic, S., Putman, M.E., Korpela, E. J,

Gibson, S. J., Begum, A., Destry, S., Robishaw, T.,

Krco, M., 2011 ApJS, 194,20

Peek J.E.G., Putman, M.E., McKee, C.F., Heiles, C., \& Stanimirović, S. 2007, ApJ, 656, 907 